\title{
Auswirkungen der Puerperalerkrankungen bei Sauen auf die Fruchtbarkeitsleistung
}

\begin{abstract}
Title of the paper: Investigations on the effects of puerperal diseases in sows on the fertility

Investigations with a total number of 1403 farrowings in one farm during 8 years have shown a frequency of puerperal diseases (MMA = mastitits, metritis, agalactiae) of $31.6 \%$ with significant differences between gilts $(41.2 \%)$ and sows (27.9 \%; $\mathrm{p}<.05)$. Gilts and sows without MMA after farrowing had a lower frequency of animals without estrus after weaning of the piglets (.2 \% vs $1.1 \%)$, a lower rate of late onset of estrus (3.4\% vs $6.2 \%$ ), a lower frequency of non-pregnant sows (16.0 \% vs $22.2 \%)$, less sows with abortion (.9 \% vs $2.2 \%)$ and a lower rate of mortality (1.1\% vs $4.0 \%)$ compared with gilts and sows with MMA. The same effects have been demonstrated both in gilts and sows.
\end{abstract}

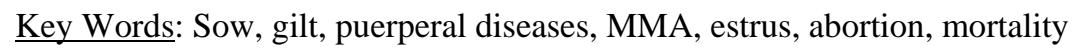

\section{Zusammenfassung}

Analysen an insgesamt 1403 Abferkelungen in einem Betrieb über insgesamt 8 Jahre ergaben eine Häufigkeit von Puerperalerkrankungen (MMA = Mastitis, Metritis, Agalaktie) von 31,6 \% mit signifikanten Unterschieden zwischen Erstlings- (41,2 \%) und Altsauen (27,9 \%; p < 0,05). Sauen ohne MMA nach der Abferkelung hatten einen niedrigeren Anteil von Tieren ohne Brunstsymptome nach dem Absetzen der Ferkel (0,2 \% vs 1,1 \%), eine geringere Rate an Spätrauschern (3,4 \% vs 6,2 \%), eine niedrigere Quote von Umrauschern (16,0 \% vs 22,2 \%), weniger Verferkelungen (0,9\% vs 2,2 \%) und eine niedrigere Zahl an Verendungen (1,1 \% vs 4,0 \%) als Vergleichstiere mit MMA. Gleichgerichtete Effekte ließen sich sowohl bei Erstlings- wie auch bei Altsauen nachweisen.

Schlüsselwörter: Altsau, Jungsau, Puerperalerkrankung, MMA, Brunst, Abort, Mortalität

Die Puerperalstörungen gehören zu den bedeutsamsten Erkrankungen der Sauen nach der Abferkelung. 30 \% der Tiere, in manchen Betrieben sogar bis 80 \% der Sauen können davon betroffen sein (HOY, 2002; PERSSON et al., 1989; WALDMANN, 2000; WENDT, 2000). Die Puerperalerkrankungen (MMA-Komplex = Mastitis, Metritis, Agalaktie) zählen zu den infektiösen Faktorenkrankheiten, bei deren Ätiopathogenese Umweltfaktoren eine große Rolle spielen (BERNER, 1984; BERTSCHINGER, 1984; HALGAARD, 1983; KAMPHUES, 2000; KOKETSU et al., 1997; STREMKE, 1980). Bei Sauen mit MMA sind die Ferkelverluste um 4 bis 5 \% höher als bei gesunden Vergleichstieren (HOY, 2002; HÜHN und REHBOCK, 1999). Darüber hinaus beeinträchtigt die Erkrankung der Mutter auch die Lebendmasseentwicklung ihrer Nachkommen. Trotz annähernd gleicher Geburtsmasse waren in den Untersuchungen von HOY (2002) die Ferkel von Sauen mit MMA am 21. Lebenstag um 3,5 \% leichter als die Stallgefährten von gesunden Müttern. Wenig war bisher darüber 
bekannt, ob die Puerperalerkrankungen auch die Fruchtbarkeitsleistung nach der folgenden Belegung beeinträchtigen können. In der Literatur liegen dazu lediglich die Untersuchungen von ROOST et al. (1988) vor, die allerdings keinen signifikanten Einfluss der MMA auf die Fertilität im Folgewurf erbrachten.

\section{Material und Methoden}

In einem Ferkelerzeugerbetrieb mit etwa 100 Sauen wurden in einem Zeitraum von acht Jahren (1995 bis 2002) sowohl die Angaben aus der Behandlungsstatistik bei den Sauen nach der Abferkelung (mit bzw. ohne Behandlung wegen MMA-Komplex) als auch die Informationen aus der Sauen-Datei (Anzahl Sauen ohne Brunst nach dem Absetzen der Ferkel, Spätrauscher, Umrauscher, Verferkelungen und Verendungen) erfasst und in einer Excel-Datei zusammengestellt. Eine Puerperalerkrankung lag bei einer Rektaltemperatur post partum von über $39,3{ }^{\circ} \mathrm{C}$ in Verbindung mit klinischen Symptomen, wie Rötung des Gesäuges und Scheidenausfluss, vor, die eine Antibiotika-Behandlung nach sich zogen. Diese Behandlungen waren bereits seit Jahren einzeltierbezogen registriert worden und ermöglichten somit die vorliegende Auswertung. Die beiden Abferkelställe des Betriebes wurden nicht nach dem Alles raus - Alles rein-Prinzip bewirtschaftet, lediglich einzelne Buchten wurden gereinigt und kontinuierlich mit hochtragenden Sauen belegt. Die Sauen standen auf einem Teilspaltenboden (ein Meter Betonboden im vorderen Teil und 1,20 m Gussroste). Nach dem Absetzen der Ferkel am durchschnittlich 28. Lebenstag wurden die Altsauen in den Besamungsstall in Einzelstände umgestallt (ebenfalls teilperforierter Boden). Bei den Altsauen erfolgte nahezu ausschließlich eine duldungsorientierte Besamung (im Mittel zwei Besamungen pro Sau). Die Jungsauen aus der eigenen Aufzucht des Betriebes wurden dagegen zumeist durch einen Eber gedeckt.

Die Excel-Datei mit den Angaben zur Wurf-Nummer der Sau, der Behandlung wegen Puerperalerkrankung nach der Abferkelung $(0=$ keine Behandlung, $1=$ Behandlung wegen MMA), zu den Daten nach dem Absetzen der Ferkel:

Auftreten der Brunst: $\quad 0=$ nein, $1=$ ja

Spätrauscher (Eintritt der Brunst

$>8$ Tage nach dem Absetzen): $\quad 0=$ nein, $1=$ ja

Umrauscher: $\quad 0=$ nein, $1=$ ja

Verferkeln: $\quad 0=$ nein, $1=$ ja

Verenden: $\quad 0=$ nein, $1=$ ja

sowie zur Wurfgröße lebend geborener Ferkel wurde in das Statistik-Programmpaket SPSS 10.0 for Windows eingelesen. Zum Vergleich der Häufigkeiten von Brunstsymptomen, Spätrauschern, Umrauschern, Verferkelungen und Verendungen in Abhängigkeit von den Puerperalerkrankungen im vorangegangenen Wurf sowie zur Prüfung der diesbezüglichen Unterschiede zwischen Jung- und Altsauen kam der Chi-QuadratUnabhängigkeitstest in Kontingenztafeln zur Anwendung. Der Mittelwertvergleich zur Wurfgröße erfolgte unter Zuhilfenahme des unabhängigen t-Tests (SPSS 10.0).

\section{3. $\quad$ Ergebnisse}

Nach den insgesamt 1403 auswertbaren Abferkelungen traten 443mal = 31,6 \% Puerperalstörungen auf. 229 Sauen schieden nach dem Absetzen der Ferkel aus der Zucht aus, so dass 1174 Probanden zur nächsten Belegung aufgestallt wurden. 
Nach den 370 Abferkelungen mit Puerperalerkrankungen hatten 1,1 \% der Sauen keine Brunstsymptome nach dem Absetzen und mussten daher gemerzt werden. Dagegen betrug dieser Anteil bei Sauen ohne MMA ( $=804$ für diese Auswertung) nach der Geburt der Ferkel lediglich 0,2 \%. Der Anteil von Spätrauschern war bei Tieren mit Puerperalstörungen mit 6,2 \% nahezu doppelt so hoch wie bei den Vergleichstieren (3,4 \%; $\mathrm{p}<0,05)$, die keine gesundheitlichen Probleme nach dem Abferkeln hatten. Die Häufigkeit von Umrauschern lag in der Gruppe von Sauen mit MMA mit 22,2 \% um 6,2 \% signifikant höher als bei denjenigen Tieren, die nach der Geburt der Ferkel weder Mastitis noch Metritis noch Agalaktie aufwiesen (16,0 \%). Sauen mit Puerperalerkrankungen in der vorangegangenen Säugezeit hatten mit 2,2 \% eine deutlich höhere Quote an Verferkelungen als Kontrollsauen ohne MMA (0,9 \%; p > 0,05). Schließlich war auch die Rate an Verendungen bei Sauen mit Puerperalstörungen nahezu viermal so hoch (4,0 \%) wie bei Sauen ohne gesundheitliche Probleme nach der Geburt ihrer Ferkel (1,1 \%; p < 0,01). Insgesamt 915 Sauen konnten bis zur nächsten Abferkelung verfolgt werden. Sauen mit MMA nach der vorangegangenen Abferkelung erzielten 0,2 lebend geborene Ferkel pro Wurf weniger als die Tiere ohne MMA. Dieser Unterschied ließ sich nicht sichern (Tab. 1).

Tabelle 1

Gesundheit und Fruchtbarkeit bei Erstlings- und Altsauen mit bzw. ohne Puerperalerkrankungen nach der vorangegangen Abferkelung (Health and fertility in gilts and sows with or without puerperal diseases after previous farrowing)

\begin{tabular}{lccc}
\hline & Sauen ohne MMA & Sauen mit MMA & Signifikanz \\
\hline $\mathrm{n}$ & 804 & 370 & \\
Anteil Sauen ohne Brunst nach dem & & & n.s. \\
Absetzen der Ferkel (\%) & 0,2 & 1,1 & $\mathrm{p}<0,05$ \\
Anteil Spätrauscher (\%) & 3,4 & 6,2 & $\mathrm{p}<0,05$ \\
Anteil Umrauscher (\%) & 16,0 & 22,2 & n.s. \\
Anteil Verferkelungen (\%) & 0,9 & 2,2 & $\mathrm{p}<0,01$ \\
Anteil Verendungen (\%) & 1,1 & 4,0 & n.s. \\
n & 655 & 260 & 10,5 \\
Wurfgröße lebend geborener Ferkel & 10,7 & &
\end{tabular}

Bei einem generell hohen Niveau der MMA-Häufigkeit in diesem Betrieb traten bei Jungsauen mit 41,2 \% wesentlich mehr Puerperalstörungen als bei den Altsauen (27,9 $\%, \mathrm{p}<0,05)$ auf (Abb.).

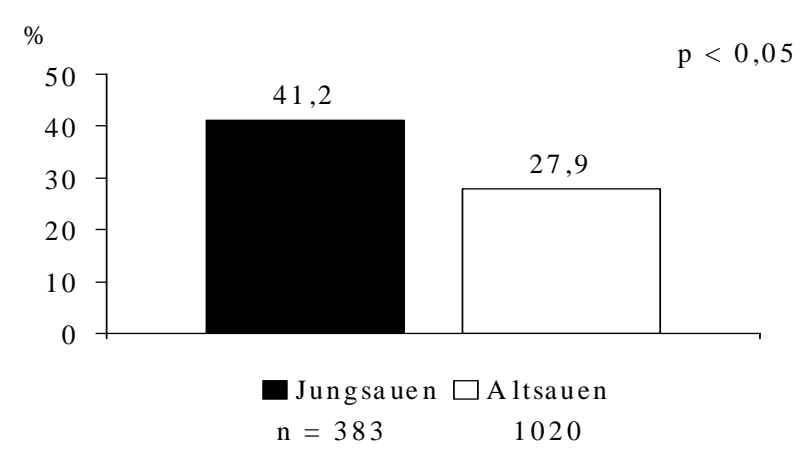

Abb.: Häufigkeit von Puerperalerkrankungen bei Erstlings- und Altsauen (Frequency of puerperal diseases in gilts and sows) 
Die nachteiligen Auswirkungen der Puerperalerkrankungen auf Gesundheit und Fruchtbarkeit nach dem Absetzen der Ferkel waren sowohl bei den Erstlingssauen wie auch bei den älteren Sauen nachweisbar. So war der Anteil von Erstlingssauen ohne Östrussymptome nach dem Absetzen des ersten Wurfes bei den Tieren mit MMA mehr als fünfmal so hoch (2,9 \%) wie bei gesunden Erstgebärenden (0,5 \%). Bei den Altsauen trat insgesamt nur ein Tier ohne Brunst nach der Trennung von den Ferkeln auf.

Der Anteil an Spätrauschern war bei Jung- und Altsauen mit gesundheitlichen Problemen nach der Abferkelung höher als bei den Stallgefährtinnen, die nicht von MMA betroffen waren. Auch die Umrauscherquote lag bei den vormals wegen Puerperalerkrankungen behandelten Jung- und Altsauen auf einem höheren Niveau im Vergleich zu den gesunden Kontrolltieren (Erstgebärende mit MMA $=+8,3 \%$; Altsauen $=+4,3 \%$ ). Ebenso hatten die Erstlings- und die Altsauen deutlich höhere Raten an Verferkelungen und Verendungen wie die Vergleichstiere ohne Erkrankung nach der Abferkelung (Tab. 2 und 3). Der Anteil der Verendungen war dabei sowohl bei den Erstlings- wie auch bei den älteren Sauen statistisch gesichert höher in der Gruppe der Tiere mit MMA. Tendenziell war auch die Wurfgröße (lebend geborene Ferkel je Wurf) im Folgewurf nach vorheriger MMA niedriger als bei nicht erkrankten Sauen.

Tabelle 2

Gesundheit und Fruchtbarkeit bei Erstlingssauen mit bzw. ohne Puerperalerkrankungen nach der vorangegangen Abferkelung (Health and fertility in gilts with or without puerperal diseases after first farrowing)

\begin{tabular}{lccc}
\hline & $\begin{array}{c}\text { Erstlingssauen ohne } \\
\text { MMA }\end{array}$ & $\begin{array}{c}\text { Erstlingssauen mit } \\
\text { MMA }\end{array}$ & Signifikanz \\
\hline $\mathrm{n}$ & 203 & 138 & n.s. \\
Anteil Sauen ohne Brunst nach dem & & & n.s. \\
Absetzen der Ferkel (\%) & 0,5 & 2,9 & n.s. \\
Anteil Spätrauscher (\%) & 5,4 & 9,5 & n.s. \\
Anteil Umrauscher (\%) & 18,7 & 27,0 & p $<0,05$ \\
Anteil Verferkelungen (\%) & 1,0 & 2,9 & n.s. \\
Anteil Verendungen (\%) & 0 & 2,9 & 88 \\
n & 162 & 9,95 &
\end{tabular}

Tabelle 3

Gesundheit und Fruchtbarkeit bei Altsauen mit bzw. ohne Puerperalerkrankungen nach der vorangegangen Abferkelung (Health and fertility in sows with or without puerperal diseases after previous farrowing)

\begin{tabular}{lccc}
\hline & Altsauen ohne MMA & Altsauen mit MMA & Signifikanz \\
\hline $\mathrm{n}$ & 601 & 232 & \\
Anteil Sauen ohne Brunst nach dem & & & n.s. \\
Absetzen der Ferkel (\%) & 0,2 & 0 & n.s. \\
Anteil Spätrauscher (\%) & 2,7 & 4,3 & n.s. \\
Anteil Umrauscher (\%) & 15,1 & 19,4 & n.s. \\
Anteil Verferkelungen (\%) & 0,8 & 1,7 & p \\
Anteil Verendungen (\%) & 1,5 & 4,7 & n.s. \\
n & 493 & 172 & 10,73 \\
Wurfgröße lebend geborener Ferkel & 10,90 & &
\end{tabular}

4. Diskussion

Mit den vorliegenden Untersuchungen konnte erneut nachgewiesen und untermauert werden, dass die Puerperalerkrankungen (MMA-Komplex) zu den wirtschaftlich 
wichtigsten Krankheiten der Sauen zählen. Nahezu ein Drittel der Sauen (31,6 \%) hatte nach der Abferkelung gesundheitliche Probleme einer Mastitis, Metritis und/oder Agalaktie mit Therapienotwendigkeit. Jungsauen (41,2 \% MMA) waren dabei signifikant stärker als Altsauen (27,9 \%) von diesem Krankheitskomplex betroffen.

Jungsauen haben einen schlechteren immunologischen Schutz als Altsauen, die sich im Laufe der Zeit schon mit mehr Krankheitserregern haben auseinandersetzen müssen (WENDT, 2000). Das Krankheitsniveau muss generell als hoch eingeschätzt werden. Primär verantwortlich dürften folgende Faktoren im Untersuchungsbetrieb sein: kontinuierliche Belegung einzelner Abferkelplätze ohne Anwendung des Alles raus - Alles rein-Prinzips für die Abferkelställe, keine Reinigung und Desinfektion der Ställe - damit keine Unterbrechung von Infektionsketten, Probleme bei der Aufstallung (Teilspaltenboden mit Gussrosten im hinteren Teil der Abferkelbuchten) und bei der Luftführung (Zugluft in einzelnen Buchten durch Vorbeiströmen kalter Luft aus dem Güllekanal und dem Stallvorraum am Hinterteil der auf den Gussrosten liegenden Sauen), besonders hohe MMA-Quoten im Winter und in bestimmten Buchten (HOY, 2002).

Mit den durchgeführten Analysen konnte gezeigt werden, dass die Puerperalstörungen nicht nur zu einem Anstieg der Ferkelverluste führen - wie u.a. in den Untersuchungen von HÜHN und REHBOCK (1999) sowie HOY (2002) nachgewiesen, sondern dass sie darüber hinaus das Brunstgeschehen nach dem Absetzen der Ferkel, die Trächtigkeitsrate bzw. Umrauscherquote sowie die Häufigkeit von Verferkelungen und Verendungen beeinflussen. In der Zusammenfassung von Erstlings- und Altsauen war bei den Tieren mit MMA nach der vorangegangenen Abferkelung der Anteil von Probanden ohne Brunstsymptome nach dem Absetzen der Ferkel sowie die Häufigkeit von Verendungen ca. vier- bis fünfmal so hoch, die Anteile von Spätrauschern und die Häufigkeit von Verferkelungen etwa doppelt so hoch und die Umrauscherquote 6,2 \% höher als bei den Vergleichstieren ohne gesundheitliche Störungen während der Säugezeit davor. Die gesundheitsbeeinträchtigenden und leistungsdepressiven Effekte des MMA-Komplexes traten - mit Blick auf den dem akuten Krankheitsgeschehen folgenden Zeitraum bis zur und nach der nächsten Belegung - sowohl bei Jung- wie auch bei Altsauen zutage. Bedingt durch die geringeren Stichprobenumfänge bei einer getrennten Auswertung von Erstlings- und älteren Sauen ließen sich die sich abzeichnenden Unterschiede jedoch mit Ausnahme der Verendungen nicht statistisch sichern. Ebenso war in der Tendenz eine um 0,2 lebend geborene Ferkel je Wurf geringere Wurfgröße im Folgewurf nach einer Puerperalstörung im Vergleich zu gesunden Sauen zu erkennen.

Mit den vorliegenden Untersuchungen war nicht das Ziel verbunden, mögliche pathophysiologische Zusammenhänge zwischen dem Auftreten der Puerperalerkrankungen und den Auswirkungen auf das Brunst- und Fruchtbarkeitsgeschehen sowie die Abgangsrate bis zur nächsten Abferkelung aufzuklären. Denkbar sind generell Störungen des Allgemeinbefindens und eine herabgesetzte Infektionsabwehrleistung der Sauen (WALDMANN, 2000; WENDT, 2000). Aus den vorgestellten Ergebnissen leiten sich jedoch die Schlussfolgerungen ab, zum einen der Hygiene bei der Bewirtschaftung der Abferkelställe eine herausragende Rolle beizumessen (Alles raus - Alles rein-Prinzip, Reinigung und Desinfektion, Stallklima- und Lüftungsgestaltung). Zum andern kommt es darauf an, Sauen mit Puerperalerkrankungen nach der Geburt sehr schnell zu erkennen und sofort zu therapieren. Unmittelbar nach der Abferkelung ist die Rektaltemperatur der Sauen zu messen, so dass bei einem Anstieg über $39,3{ }^{\circ} \mathrm{C}$ die 
betreffenden Sauen zügig mit Antibiotika behandelt werden können (WALDMANN, 2000). Es ist bekannt, dass eine sofortige Therapie im Vergleich zu einer 7 bis 24 Stunden zu spät durchgeführten Antibiotika-Applikation ein deutliches Ansteigen der Ferkelverluste verhindert (LUTTER und SCHULZ, 1984). Im Analogieschluss ist zu postulieren, dass die möglichst frühe therapeutische Versorgung von Sauen mit Puerperalerkrankungen eine günstige Prognose auch im Hinblick auf die späteren Gesundheits- und Leistungsdaten der vormals erkrankten Tiere erwarten lässt.

\section{Danksagung}

Carmen Weirich wird für die technische Mitarbeit an diesen Untersuchungen gedankt.

BERNER, H.:

\section{Literatur}

Die Bedeutung von Harnwegsinfektionen für die Entstehung der puerperalen Endometritis beim Schwein. Tierärztl. Umschau 39 (1984), 450-458

BERTSCHINGER, H.U.:

Neue Aspekte der Pathogenese der puerperalen Mastitis. Tierärztl. Umschau 39 (1984), 458-461

HALGAARD, CH.:

HOY, ST.:

Epidemiologic factors in puerperal diseases of sows. Nord. Vet.-Med. 35 (1983), 161-174

Untersuchungen zum Einfluss verschiedener Haltungsfaktoren auf die Häufigkeit von Puerperalerkrankungen bei Sauen. Praktischer Tierarzt 83 (2002) 11, 990-996

HÜHN, U.; REHBOCK, F.:

Prostaglandine contra Umrauschen. Krankhaftem Scheidenausfluss nach der Geburt Paroli bieten. dlz agrarmagazin 50 (1999), 192-196

KAMPHUES, J.:

MMA: Den Darm auf Touren halten. top agrar 2 (2000), S8-S10

KOKETSU, Y.; DIAL, G.D.; KING, V.L.:

Returns to service after mating and removal of sows for reproductive reasons from commercial swine farms. Theriogenology 47 (1997), 1347-1363

LUTTER, K.; SCHULZ, B.:

Zur Senkung der durch Aufzuchterkrankungen verursachten wirtschaftlichen Verluste mittels frühzeitiger Behandlung. Tierzucht, Berlin 38 (1984), 553-554

PERSSON, A.; PEDERSEN, A.E.; GÖRANSSON, L.; KUHL, W.:

A long term study on the health status and performance of sows on different feed allowances during late pregnancy. 1. Clinical observations, with special reference to agalactia post partum. Acta vet. scand. 30 (1989), 9-17

ROOST, H.; KUNTZSCH, G.; BERBER, H.; BLEY, G.; HÜHN, U.:

Untersuchungen zum Einfluss von Puerperalerkrankungen auf die Fruchtbarkeits- und Aufzuchtleistungen in einer industriemäßig produzierenden Sauenanlage. Mh. Vet.-Med. 43 (1988), 197-200

STREMKE, A.:

Untersuchungen über die Fruchtbarkeitsleistungen von ovulationssynchronisierten Sauen nach Verkürzung der vorangegangenen Säugezeit auf unter 4 Wochen. AdL Berlin, Diss. A, 1980

WALDMANN, K.-H.:

Behandeln Sie MMA-Probleme rechtzeitig. top agrar 3 (2000), S4-S7

WENDT, M.:

So optimieren Sie das Geburtsmanagement. top agrar 1 (2000), S6-S8

Eingegangen: 17.12.2002

Akzeptiert: 07.05.2003

Autor für Korrespondenz

Prof. Dr. STEFFEN HOY

Justus-Liebig-Universität Gießen, Institut für Tierzucht und Haustiergenetik

Bismarckstraße 16

D-35390 Gießen 